\title{
The Dominant Influence of Plastic Deformation Induced Residual Stress on the Barkhausen Effect Signal in Martensitic Steels
}

\author{
Leszek Piotrowski $^{1,2}$. Marek Chmielewski ${ }^{2} \cdot$ Zbigniew L. Kowalewski $^{3}$
}

Received: 22 September 2016 / Accepted: 20 December 2016 / Published online: 2 January 2017

(C) The Author(s) 2017. This article is published with open access at Springerlink.com

\begin{abstract}
The paper presents the results of investigation of the influence of plastic deformation on the magnetic properties of martensitic steel (P91 grade). The properties of the hysteresis loops as well as of the Barkhausen effect (BE) signal are analysed for both tensile and compressive loading up to $\varepsilon=10 \%$ of plastic deformation. The choice of the steel and of the deformation range is unique, since for such combination one can expect high residual stresses (both compressive and tensile) in the material that does not exhibit saturation of the BE intensity as a function of elastic stress. The obtained relationships show that for the low level of deformation the dislocation density changes may play a dominant role, yet for higher deformation level the residual stress becomes a dominant factor. It leads to the strong decrease of the BE signal for tensile deformation and an increase for the case of compression. It agrees well with the assumption that the tensile plastic deformation results in the compressive stresses appearance in the soft (magnetically active) subregions of the material whereas for the compression one can expect a residual stress of a tensile nature in those areas. Both deformation modes result in the increase of coercivity of the samples, yet the increase observed for the tensile deformation is significantly higher since both the residual compressive stress and increase of dislocation density have a strong effect on the material coercivity. The change of the
\end{abstract}

Leszek Piotrowski

lesio@mif.pg.gda.pl

1 Faculty of Applied Physics and Mathematics, Gdansk University of Technology, Narutowicza 11/12, 80-233 Gdańsk, Poland

2 Physics Department, Gdansk University of Technology, 80-233 Gdańsk, Poland

3 Institute of Fundamental Technological Research, 02-106 Warsaw, Poland hysteresis loops steepness agrees well with the notion of the dominant role of residual stresses too.

Keywords Barkhausen effect - Plastic deformation · Residual stress · Magnetic hysteresis · Coercivity

\section{Introduction}

The process of plastic deformation results in a strong modification of the dislocation density and arrangement. At the beginning of the process there is a sharp increase of the density of randomly distributed dislocations that for the higher deformation levels tend to group and form the so called dislocation cell structure. Such structure consists of the hard (both mechanically and magnetically) regions of the cell walls and much softer (with lower dislocation densities) cell interiors. In such structures there appear internal residual stresses of opposite signs in soft and hard regions. For instance after the tensile deformation, in the unloaded state, the soft regions are being compressed while the hard ones remain under tension [1-3]. The dislocation tangles create not only the obstacles hindering the plastic flow of the material but also tend to interact strongly (in the ferromagnetic materials) with the magnetic domain walls (DW). The discontinuous motion of the DWs that are pinned by dislocation tangles (but also precipitates, grain boundaries and other material inhomogeneities) results in the local abrupt change of the magnetic flux density that can be detected with the help of the detecting coil, either encircling the investigated material or placed close to its surface. Such effect is called the Barkhausen effect (BE) and the induced voltage is referred to as the Barkhausen noise signal. One has, however, to take into account that only the signal from the close to the surface region can be detected, since the BE signal is strongly attenuated in con- 
ducting materials (due to its relatively high frequency of order of up to about $100 \mathrm{kHz}$ or even higher). Because the direct source of the BE are the processes of interaction of DWs with the microstructural features, the BE noise signal is closely related to the dislocation density and arrangement. Being so, it should be possible to apply the analysis of the BE signal properties for the assessment of the plastic deformation level in ferromagnetic materials. Such attempts have been made yet, as it turned out, the observed dependence of the BE signal intensity on the deformation level is strongly material dependent-for the tensile deformation it can increase [4] for soft materials, such as e.g. Armco iron, decrease [5] for high strength martensitic steels, or behave in a nonmonotonic way $[6,7]$ for the intermediate materials. Being so the assessment of the deformation level sometimes requires application of rather advanced signal analysis techniques. Most studies were carried out on the samples subjected to tensile deformation due to serious problems with the buckling of the samples during the compression. For the mechanical test the problem can be avoided by an appropriate sample geometry, yet samples of such shape are not well suited for the BE measurements. Nonetheless an attempt on such measurements has also been made by Kleber and Vincent [8]. Even though the compression level was significantly lower than in the case of tensile deformation, on the basis of the combined analysis of the BE signal intensity and shape they suggested that the problem with prediction of the BE signal intensity behaviour during deformation arises from the fact that it is a result of two factors - the change in the dislocation density and internal stresses distribution. The internal stress level of the higher order is directly connected with the dislocation density, yet the influence of them on the BE intensity is different. Such statement was to a high degree intuitive as they investigated relatively soft materials (with low residual stress level expected). In our paper we analyse the behaviour of high strength steel subjected to high compression level and the results fully confirm that notion.

Apart from the changes in the BE signal properties the "normal" magnetic properties, such as the hysteresis loop shape or coercivity, also change during the plastic deformation $[9,10]$. The increase in the dislocation density leads to the increase in the coercivity of the material for both compressive and tensile deformation. In addition to that a characteristic "bulge" is observed for the samples subjected to tensile deformation. The appearance of such feature is believed to be the result of the appearance of regions of high compressive residual stress in the areas with lower dislocation densities such as dislocation cells interiors [11]. Such changes, even though they are most probably a signature of plastics deformation cannot be usually applied for the assessment of the plastic deformation level in a non-destructive way in industrial components since the hysteresis measurements are not easily performed in such conditions. The sensitivity of magnetic parameters may be improved by the application of Magnetic Adaptive Technique (MAT) [12], the method, however, requires somewhat complicated data processing to find the optimum signal descriptors.

The paper presents unique data as is analyses martensitic steel subjected to both compressive and tensile deformation of high level. Due to its microstructure, which is very fine grained and characterized by the high dislocation density, the dependence of the BE intensity on the stress level is very strong and does not tend to saturate in the elastic regime [13] (which is the case for ferritic/bainitic steels). In addition to that, thanks to the high yield strength of the samples one can expect important back stresses arising as a result of plastic deformation. As a result of those two facts one can expect the residual stress to play a dominant role in the behaviour of the BE signal for strongly deformed samples.

\section{Experimental}

A set of samples made of P91 high chromium martensitic steel (chemical composition given in Table 1) subjected to tensile and compressive deformation was tested. The obtained deformation levels (relative elongation measured after unloading) are given in Table 2.

In addition to that, a non-deformed sample ( $\mathrm{S} 0$ ) was investigated. The samples subjected to the compression were twice as numerous as the ones after tensile deformation due to the possibility of errors during their preparation-which luckily did not occur. The shape of the samples is shown in Fig. 1. As can be seen, the gauge length of the sample is rather low compared to the total sample length. It was necessary in order to use the specially designed anti-buckling fixture, the detailed description of which can be found in the paper by Ditrich et al. [14]. Application of such fixture was a key solution necessary in order to obtain highly compressed samples of low width in which the ratio of the BE signal intensity to the signal generated by the rate of change of magnetic flux in the bulk of material is adequately high. The schematic view of the measurement set is shown in Fig. 2. The sample (1) was placed on the yoke (4) made of electrical steel that was used to minimize demagnetisation and to magnetise only the interesting part of the samples. The size of the yoke was chosen to fit the gauge length of the sample in order to minimize the influence of the non-deformed parts of the sample on the results of magnetic measurements. The sample was magnetized with the help of the encircling coil (2) with number of turns $n_{m a g}=300$ and a smaller encircling coil (3), for which $n_{\text {sense }}=200$, was used for the BE signal detection. The magnetising frequency was about $0.5 \mathrm{~Hz}$ for the $\mathrm{B}(\mathrm{H})$ measurements (for the BE measurements the same slope of the magnetising current was applied). 
Table 1 Chemical composition of P91 steel

\begin{tabular}{lllllllllllllll}
\hline Element & $\mathrm{C}$ & $\mathrm{Si}$ & $\mathrm{Mn}$ & $\mathrm{P}$ & $\mathrm{S}$ & $\mathrm{Cr}$ & $\mathrm{Mo}$ & $\mathrm{Ni}$ & $\mathrm{Al}$ & $\mathrm{Cu}$ & $\mathrm{Ti}$ & $\mathrm{Nb}$ & $\mathrm{V}$ \\
\hline Wt. (\%) & 0.085 & 0.27 & 0.30 & 0.015 & $<0.01$ & 8.2 & 0.86 & 0.16 & 0.01 & 0.15 & $<0.01$ & 0.098 & 0.19 \\
\hline
\end{tabular}

Table 2 Deformation levels of the investigated samples

\begin{tabular}{|c|c|c|c|c|c|c|c|c|c|c|}
\hline & $\mathrm{T} 1$ & $\mathrm{~T} 2$ & $\mathrm{~T} 3$ & $\mathrm{~T} 4$ & T5 & T6 & $\mathrm{T} 7$ & $\mathrm{~T} 8$ & T9 & $\mathrm{T} 10$ \\
\hline \multicolumn{11}{|c|}{ Tensile deformed samples } \\
\hline \multirow[t]{2}{*}{$\varepsilon(\%)$} & 0.102 & 0.195 & 0.294 & 0.393 & 0.487 & 0.793 & 1.593 & 2.484 & 5.01 & 9.971 \\
\hline & $\mathrm{C} 1$ & $\mathrm{C} 2$ & $\mathrm{C} 3$ & $\mathrm{C} 4$ & C5 & C6 & $\mathrm{C} 7$ & $\mathrm{C} 8$ & $\mathrm{C} 9$ & $\mathrm{C} 10$ \\
\hline \multicolumn{11}{|c|}{ Compressed samples } \\
\hline \multirow[t]{2}{*}{$\varepsilon(\%)$} & 0.096 & 0.198 & 0.306 & 0.402 & 0.487 & 0.801 & 1.597 & 2.486 & 4.983 & 10.03 \\
\hline & $\mathrm{C} 11$ & $\mathrm{C} 12$ & $\mathrm{C} 13$ & $\mathrm{C} 14$ & $\mathrm{C} 15$ & $\mathrm{C} 16$ & $\mathrm{C} 17$ & $\mathrm{C} 18$ & C19 & $\mathrm{C} 20$ \\
\hline \multicolumn{11}{|c|}{ Compressed samples } \\
\hline$\varepsilon(\%)$ & 0.106 & 0.201 & 0.314 & 0.409 & 0.503 & 0.801 & 1.600 & 2.501 & 5.010 & 10.09 \\
\hline
\end{tabular}

Fig. 1 The shape of the investigated samples (dimensions in $\mathrm{mm}$ )

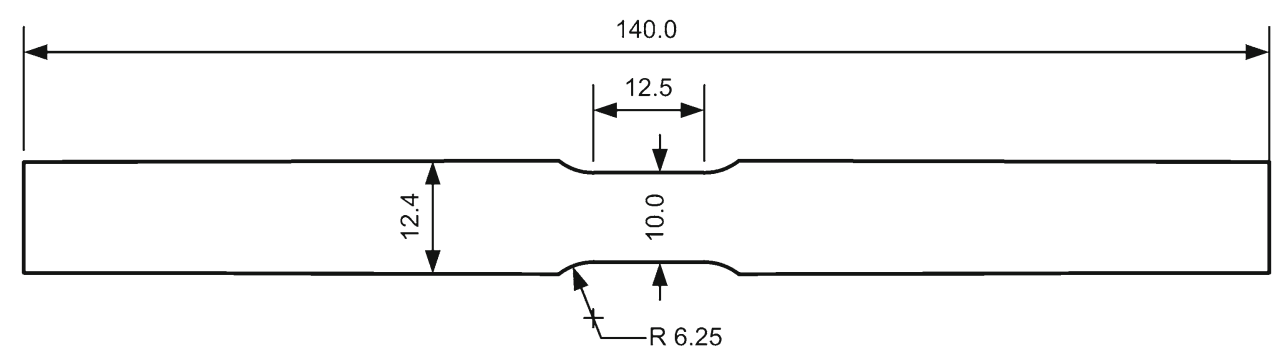

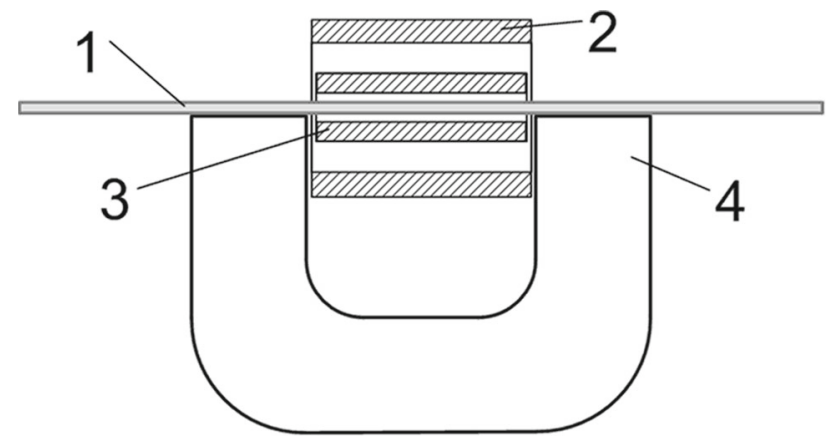

Fig. 2 Schematic view of the measurement set-up. 1 sample, 2 magnetising coil, 3 sensing coil, 4 soft magnetic yoke

Both coils were dedicated to the measurement setup (their carcasses were prepared using 3D printing) and fitted the gap between the yoke legs in order to make the best use of the sample gauge length. The magnetising coil was fed by the triangular in form current from the analog current amplifier driven by the signal provided by the multifunction data acquisition device NI USB-6366. The same device was also responsible for the signal acquisition (sampling rate was equal to $2 \mathrm{MHz}$ ). The signal from the detecting coil was pre-amplified and then either used for the hysteresis loops determination (numerical integration) or after the low fre- quency component removal (HPF filtering, $f_{\text {cut }- \text { off }}=800$ $\mathrm{Hz}$ ) and further amplification treated as the BE noise signal. The as-measured BE signal was numerically filtered (1-300 $\mathrm{kHz}$ ) and its rms-like envelopes and pulse count distributions were recorded.

\section{Results}

\subsection{Magnetic Properties}

The hysteresis loops obtained for the samples subjected to the tensile and compressive deformation are shown in Figs. 3 and 4 respectively. For the tensile deformation, the behaviour is typical and agrees well with the results described in literature. As the deformation progresses the coercivity increases, the steepness of the loops decrease and characteristic "bulge" in the central part of hysteresis loop appears. As it was mentioned earlier such deformation of the central part of the loop is probably due to the residual compressive stresses after the unloading of the sample. Such assumption is confirmed by the fact that it is not observed for the plastically deformed samples (tensile deformation) that were investigated in the loaded state [15]. Such shape deformation can be also mod- 


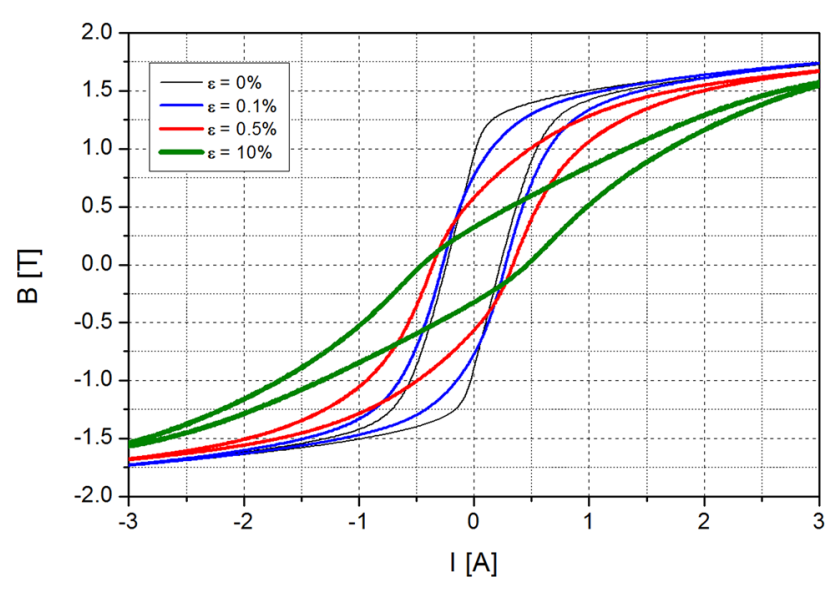

Fig. 3 Magnetic hysteresis loops for the samples subjected to tensile deformation

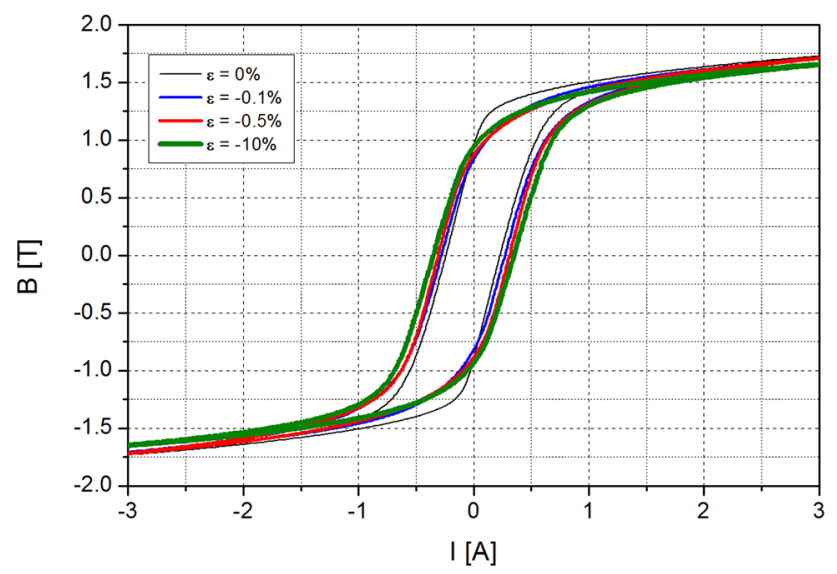

Fig. 4 Magnetic hysteresis loops for the samples subjected to compressive deformation

elled with the help of Preisach simulations in the material subjected to compressive stress [16]. For the samples subjected to compression the loops do not undergo such a serious modification. The coercivity increases during deformation, yet there is no pronounced change in the steepness of the obtained loops. It is understandable if we assume the presence of the residual stresses of tensile type, such stresses can reach relatively high level for martensitic steels [17]. It is known that such stresses do not modify the shape of hysteresis loops very much (see e.g. [18]), so the most important factor influencing the hysteresis loop change becomes the increase of dislocation density which is strongly correlated with the coercivity of the samples.

The quantitative characterisation of the observed loops is given in Fig. 5 and Fig. 6 in which the coercivity and differential permeability (maximum values) are plotted (relative values, with the reference value being the one obtained for the non-deformed sample). As for the coercivity it is clearly seen that both deformation modes result in its increase, yet for the tensile deformation the increase is twice as high as in

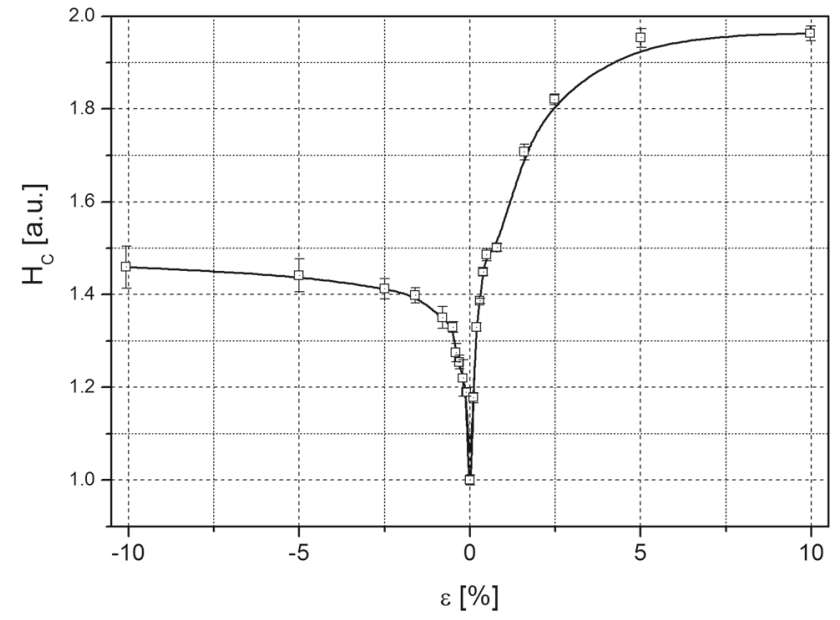

Fig. 5 Coercivity of the investigated samples as a function of the deformation level

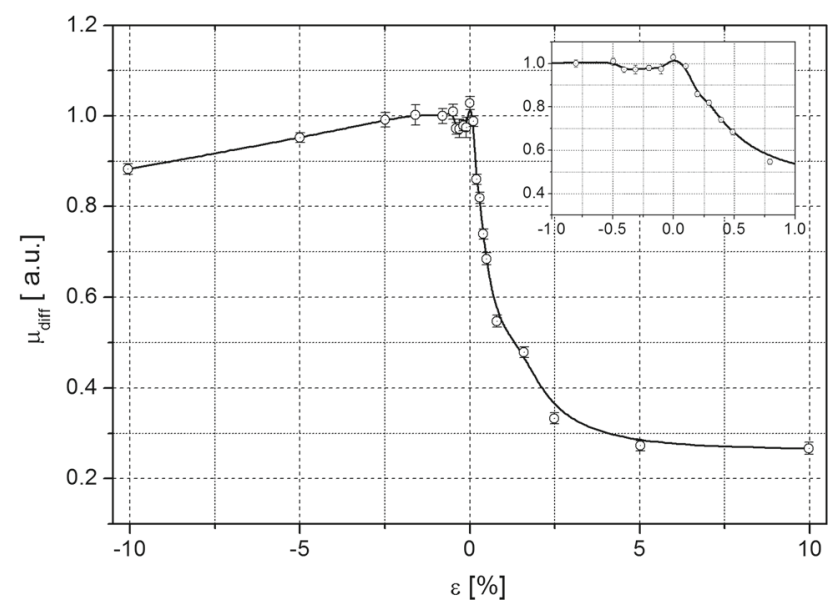

Fig. 6 Differential permeability of the investigated samples as a function of the deformation level

the case of compression. It is in agreement with the fact that for tensile deformation both the compressive residual stress and increasing dislocation density tend to increase coercivity. On the other hand, for compression we expect the tensile residual stress, the influence of which on the coercivity is very small $[19,20]$. The observed changes of differential permeability (characterizing the steepness of hysteresis loops) are very strong only for the tensile deformation for which the total decrease (for high deformation levels) exceeds $70 \%$. For the compression the changes are very small-the initial strong dislocation density increase causes a small decrease of permeability, then it is followed by a slight increase and finally it starts to decrease (by about $10 \%$ for the highest deformation level). As can be seen, by analysing both parameters (coercivity and permeability) it is possible to assess the deformation level despite the fact the coercivity increases for both deformation modes. 


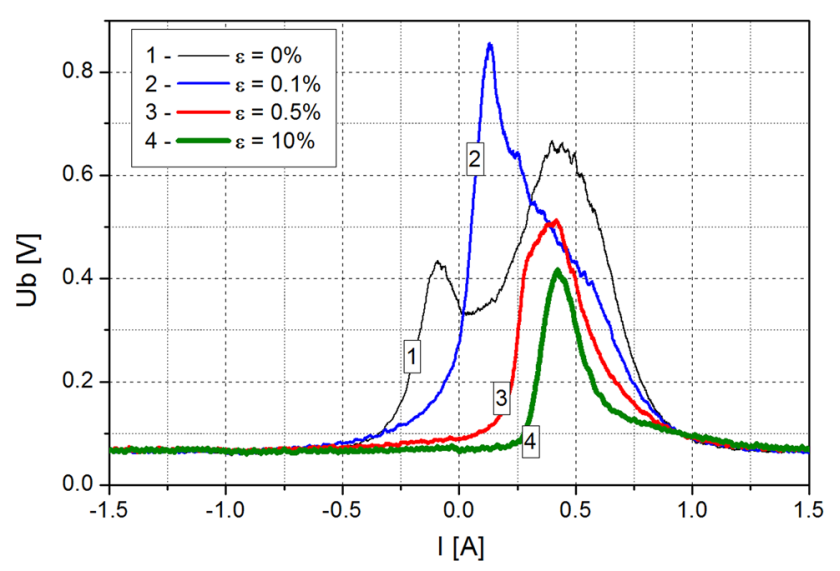

Fig. 7 Barkhausen effect signal envelopes for samples subjected to tensile deformation

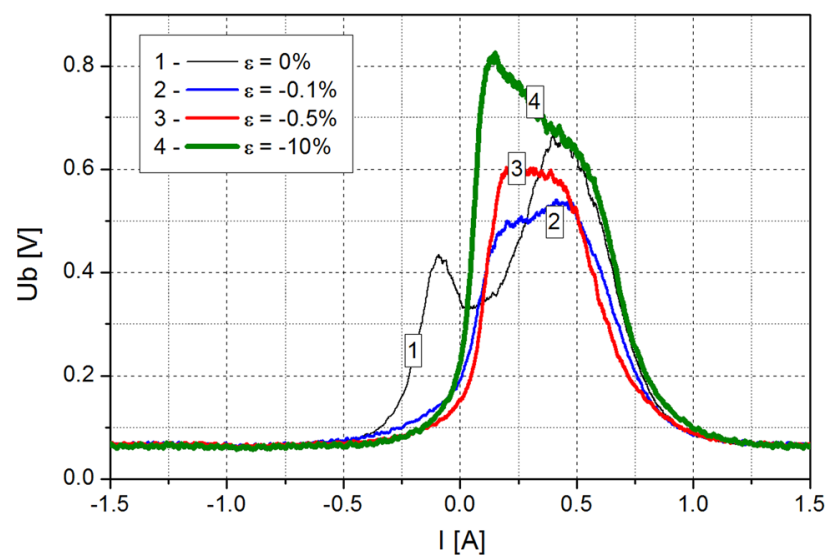

Fig. 8 Barkhausen effect signal envelopes for samples subjected to compression

\subsection{Barkhausen Effect Signal Properties}

The rms-like envelopes (plotted as a function of the magnetising current intensity) of the Barkhausen noise signal are shown in Fig. 7 (tensile deformation) and Fig. 8 (compression). The magnetisation range is somewhat lower than in the case of $\mathrm{B}(\mathrm{H})$ measurements $\left(I_{M A X}=1.65 \mathrm{~A}\right)$ since it was observed that the higher magnetising current did not result in an observable change of the BE signal. For the compressed samples the range was high enough to magnetically saturate the samples, but even for the samples after tensile deformation, for which the induction reached only about $1 \mathrm{~T}$ for the magnetically hardest sample, all the detectable BE events occured before reaching the maximum of the applied magnetizing current. As can be seen, the shape of the envelopes changes very strongly for both modes of deformation right from the start of the process. The initially observed two peak structure disappears, even for the samples with the smallest level of plastic deformation. In the case of tensile deformation the amplitude initially strongly increases, yet as the deforma- tion progresses it starts to decrease. It may be explained by the strong increase in the dislocation density and creation of dislocation tangles at the onset of deformation. Such tangles create additional pinning sites making the DWs movement more intermittent and resulting in the increase of the $\mathrm{BE}$ intensity for the field region where such pinning/depinning processes are most intensive. As the deformation progresses the dislocation tangles structure rearranges and dislocation cell structure is formed in which the prevailing number of dislocations is concentrated in the cell walls while the cell interiors are relatively free from dislocations. In such a structure the strongest BE activity is due to the DW movement inside the cells. Since those regions, after tensile deformation, are subjected to compressive residual stresses, the BE signal intensity becomes smaller due to the decreasing activity of $180^{\circ} \mathrm{DWs}$ being the main source of the BE signal $[21,22]$. The other characteristic feature of the BE signal envelopes is the shift of their maxima toward higher field values for the more deformed samples. It is in a good agreement with the fact that the observed BE intensity should correlate with the coercivity, as it is the field region where the most events of pinning/unpinning of DWs take place. It is not always so since there is also another mechanism of the BE signal generation, namely the DWs creation/annihilation processes that take place for the field regions for which the "knees" of hysteresis loops are observed [4]. For the case of compression the changes are qualitatively different, there is no increase of the signal amplitude at the onset of the process (to the contrary-it decreases), but during the deformation progress such increase is clearly seen. Such behaviour may arise from the fact that martensitic steels in the as-delivered condition possess rather high dislocation density resulting in significant level of compressive residual stress of higher order. Being so one cannot expect the dislocation structure modification to by "symmetrical" for both modes of deformation. As for the further increase of the BE signal for higher deformation levels it confirms the assumption that the residual stress of tensile type becomes the dominant factor.

Quantitatively the BE signal intensity changes are shown in Fig. 9, where the $\operatorname{Int}(\mathrm{Ub})$ parameter is an integral of the BE signal envelope over half-period of magnetisation (calculated after the background noise level subtraction). The observed relationship displays two characteristic featureson the smooth sigmoidal curve there is superimposed a sharp peak observed for low deformation level. The sigmoidal curve resembles strongly typical curves representing the dependence of BE signal intensity on the elastic strain level, that can be observed for many different materials [22]. Naturally one has to take into account the fact that the curves are reversed, since the positive plastic deformation results in negative compressive stresses and vice versa. As for the central peak, in this region the overall BE intensity decreases for both deformation modes (even though the amplitudes behave in a 


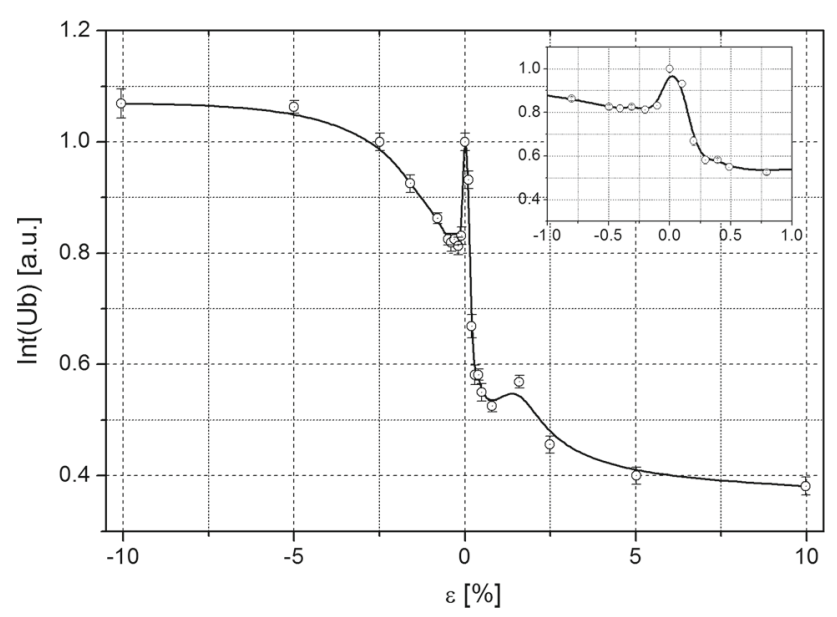

Fig. 9 BE signal intensity as a function of the deformation level

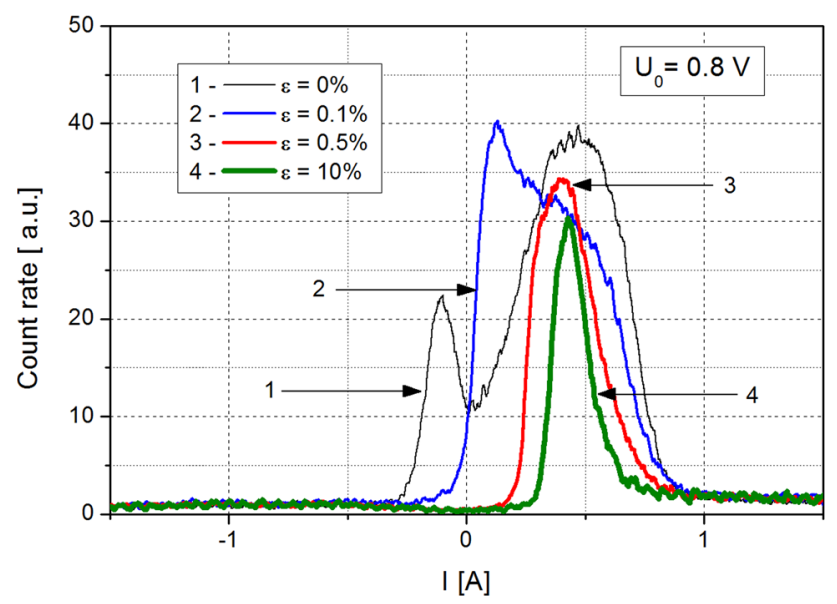

Fig. 10 Pulse count rate - tensile deformation (low threshold voltage)

different way) due the dislocation structure modificationthe changes in that region may be material dependent due to the differences in the dislocation structure of their initial state [4-7]. For the higher deformation levels the dislocation structure modification becomes less significant and being so the sigmoidal curve can be treated as a curve obtained for the material with high dislocation density subjected to elastic stress.

Another approach to the BE signal analysis, especially useful for the elastic strain level assessment is the pulse count analysis [21]. The plots presenting the pulse count rate as a function of magnetising current are shown for low $\left(U_{0}=0.8 \mathrm{~V}\right)$ threshold voltage (voltage level above which the pulses are counted) in Fig. 10 (tensile deformation) and Fig. 11 (compression). The observed behaviour is similar to that observed for the rms-like envelopes but the changes are less pronounced what suggests that the changes in the signal intensity are mostly due to the high voltage pulses. Pulse count rate for low threshold level treats high and low pulses in

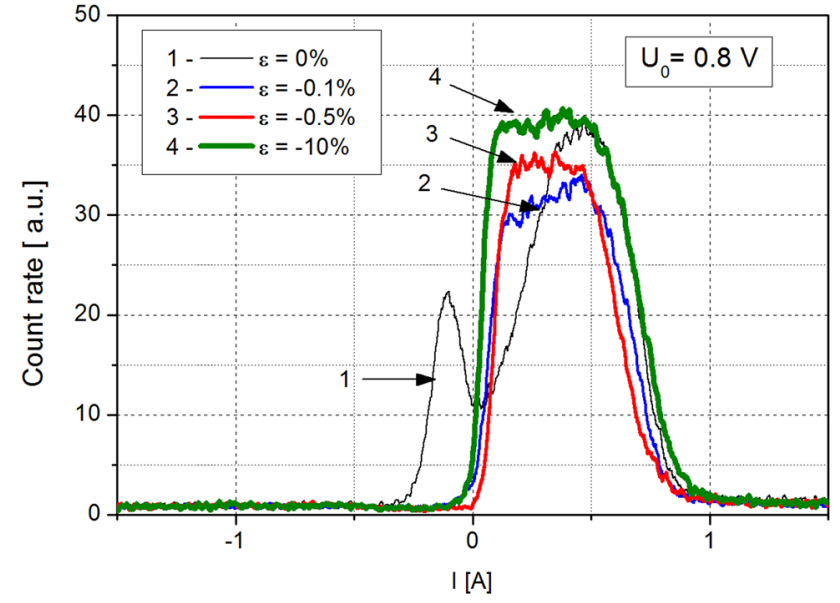

Fig. 11 Pulse count rate-compression (low threshold voltage)

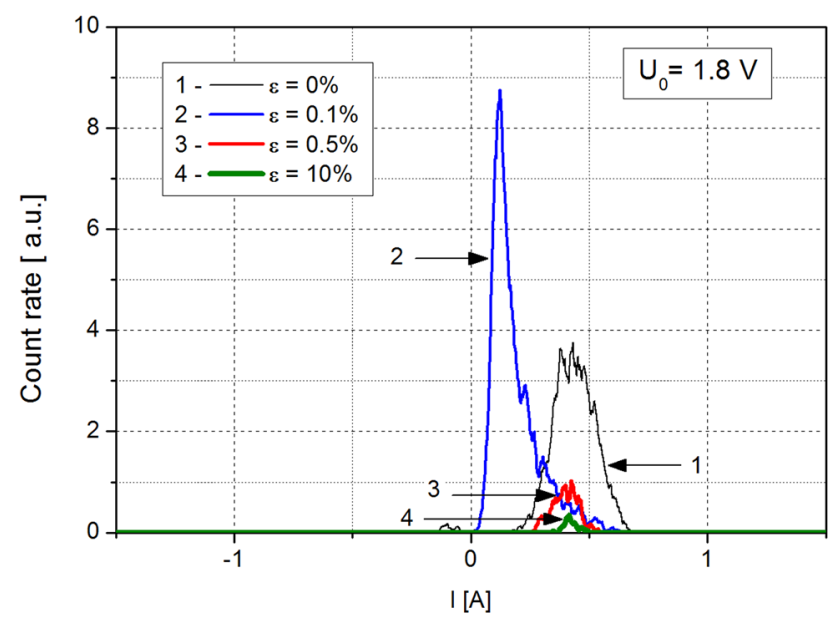

Fig. 12 Pulse count rate — tensile deformation (high threshold voltage)

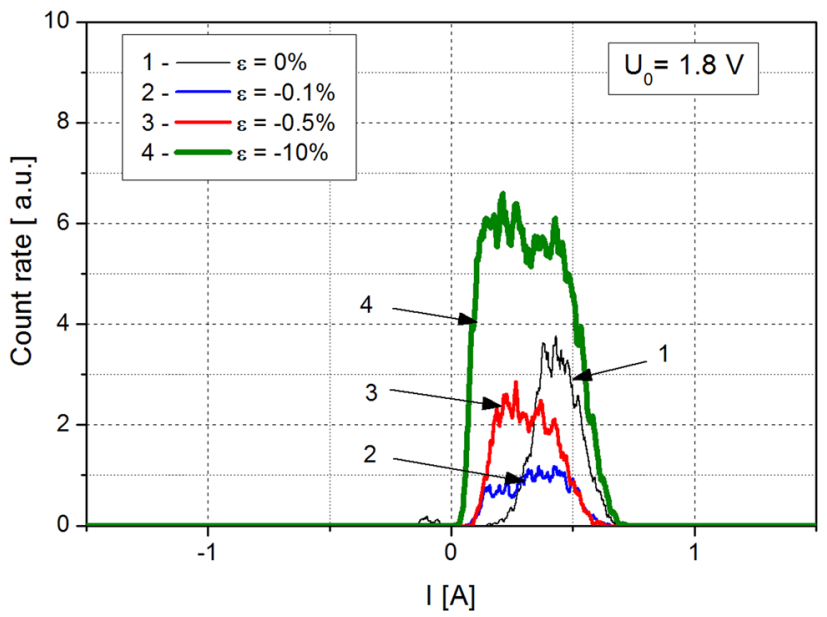

Fig. 13 Pulse count rate-compression (high threshold voltage)

the same way hence the influence of the first ones on the signal intensity is underestimated. Two following figures present the analogous results obtained for the high $\left(U_{0}=1.8 \mathrm{~V}\right)$ 


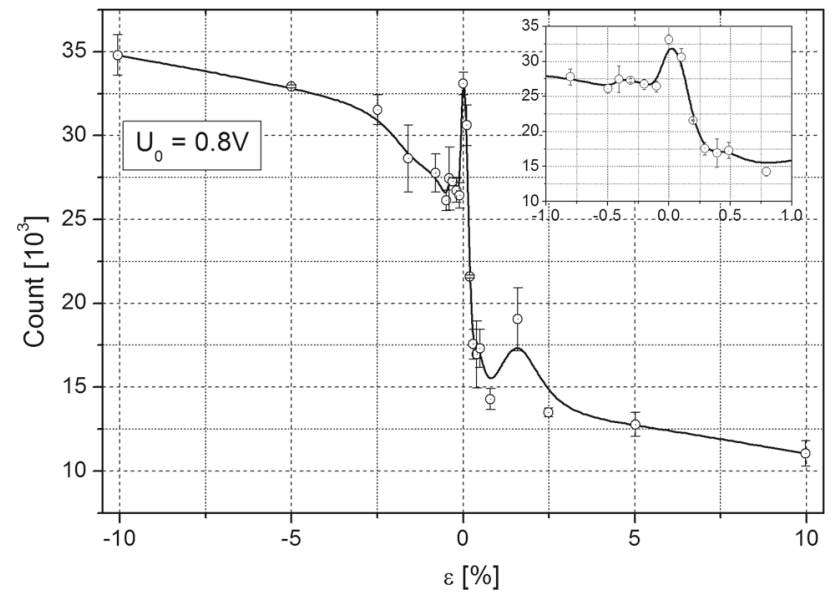

Fig. 14 Total pulse count as a function of deformation level-low threshold voltage $U_{0}=0.8$

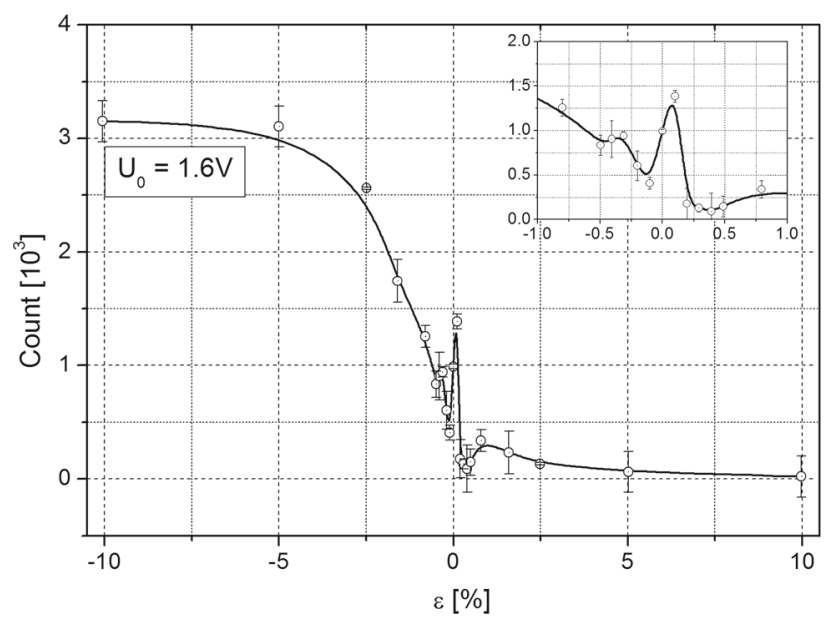

Fig. 15 Total pulse count as a function of deformation level-low threshold voltage $U_{0}=1.8 \mathrm{~V}$

threshold voltage level (Fig. 12 shows the results for tensile deformation, and Fig. 13 for compression). In this case the observed differences are much more pronounced. The increase in the maximum count rate for the low level of tensile deformation is very strong-it is over two times higher than for the non-deformed sample. It shows that the high pulses, resulting from the abrupt depinning from newly created dislocation tangles are the main source of the $\mathrm{BE}$ intensity envelope maximum increase. As for the compressive deformation, the changes are non-monotonic, the count rate initially decreases, but as the deformation progresses the observed increase is much more pronounced than the one observed for the small pulses. One can also observe that for the high threshold level and for the non-deformed sample the first peak (clearly visible while analysing small pulses) almost disappears. For the purpose of the quantitative analysis we used as a parameter the total pulse count. The plots showing the dependence of the total pulse count on the deformation level are shown in Fig. 14 (low threshold voltage level $U_{0}=0.8 \mathrm{~V}$ ) and Fig. 15 (high threshold voltage level $U_{0}=1.8 \mathrm{~V}$ ). Both curves have the shape similar to the one observed for the curve plotted in the case of the $\mathrm{BE}$ intensity analysis, but the range of the relative change of the sigmoidal function is much higher in the case of the high threshold level than in the case of the lower one. It is a very important result as such behaviour is one of the most characteristic features of the curves obtained during the calibration procedure (pulse count as a function of elastic strain) of various steels [21]. The range of change obtained, during such procedure, is always much higher while using the high threshold. The only problem with application for the analysis of elastic strain (and also, as can be seen, plastic deformation) of the calibration curves based on the high pulse total count is the fact that it may become too small and hence prone to high statistical scatter. In our case, for instance the count for the non-deformed sample is about thirty times lower for high threshold than for the low one. The difference is even bigger for the highest tensile deformation level, in which case the count for high threshold decreases to merely 22 pulses (about 500 times lower than for the low threshold) what does not guarantee obtaining reliable results. What is interesting, also for the tensile deformation there is observed local maximum (for the $\varepsilon=1.6 \%$ ) which is probably due to the dislocation cell formation onset.

\section{Conclusions}

The unwanted plastic deformation process is one of the potentially most dangerous causes of industrial components failure. Being so, the possibility of nondestructive evaluation of the plastic deformation level has been investigated for a long time now. The properties of the magnetic hysteresis loops change strongly during plastic deformation and by using two parameters (e.g. permeability and coercivity) that level could be determined unambiguously. Such measurements, however, are usually not feasible in the industrial environment. The obvious alternative seems to be application of the Barkhausen effect signal analysis. Yet, even though the possibility had been investigated for many years, the results were not obvious and various trends were observed. It was suggested that such discrepancy may be due to the fact that two different processes have to be taken into account. First one is the dislocation density and distribution modificationthe process that is strongly dependent on the material initial dislocation structure. The second one is the appearance of the residual stresses of the character opposite to the applied deformation (compressive stress for plastic tensile deformation). This process leads always to the increase of the $\mathrm{BE}$ intensity for the compressive deformation and its decrease for the tensile one. The strength of the change depends on 
the level of residual stress and hence is connected with the material yield strength (the stronger the material the higher residual stress may be expected).

Our results, showing the sigmoidal dependence of the BE signal intensity on the plastic deformation level fully confirm the importance of the residual stress. In addition to that, the very characteristic feature deduced from the pulse count analysis - namely the stronger rate of change of the count rate of higher pulses-further confirms the assumption that the residual stress is the main factor governing the observed $\mathrm{BE}$ intensity changes for higher deformation levels in high strength martensitic steels.

Acknowledgements This work has been partially funded by the Polish National Science Centre grant No 2014/15/B/ST8/04368.

Open Access This article is distributed under the terms of the Creative Commons Attribution 4.0 International License (http://creativecomm ons.org/licenses/by/4.0/), which permits unrestricted use, distribution, and reproduction in any medium, provided you give appropriate credit to the original author(s) and the source, provide a link to the Creative Commons license, and indicate if changes were made.

\section{References}

1. Feaugas, X.: On the origin of the tensile flow stress in the stainless steel AISI $316 \mathrm{~L}$ at $300 \mathrm{~K}$ : back stress and effective stress. Acta Mater. 47, 3617-3631 (1999). doi:10.1016/ S1359-6454(99)00222-0

2. Mughrabi, H.: Deformation-induced long-range internal stresses and lattice plane misorientations and the role of geometrically necessary dislocations. Philos. Mag. 86, 4037-4054 (2006). doi:10. 1080/14786430500509054

3. Mughrabi, H.: Dual role of deformation-induced geometrically necessary dislocations with respect to lattice plane misorientations and/or long-range internal stresses. Acta Mater. 54, 3417-3427 (2006). doi:10.1016/j.actamat.2006.03.047

4. Piotrowski, L., Augustyniak, B., Chmielewski, M., Hristoforou, E.V., Kosmas, K.: Evaluation of Barkhausen noise and magnetoacoustic emission signals properties for plastically deformed Armco iron. IEEE Trans. Magn. 46(5), 239-242 (2010). doi:10. 1109/TMAG.2009.2034020

5. Makowska, K., Kowalewski, Z.L., Augustyniak, B., Piotrowski, L.: Determination of mechanical properties of P91 steel by means of magnetic Barkhausen emission. J. Theor. Appl. Mech. 52, 181-188 (2014)

6. Stefanita, C.G., Atherton, D.L., Clapham, L.: Plastic versus elastic deformation effects on magnetic Barkhausen noise in steel. Acta Mater. 48, 3545-3551 (2000). doi:10.1016/ S1359-6454(00)00134-8

7. Piotrowski, L., Augustyniak, B., Chmielewski, M., Kowalewski, Z.: Multiparameter analysis of the Barkhausen noise signal and its application for the assessment of plastic deformation level in 13HMF grade steel. Meas. Sci. Technol. 21, 115702 (2010). doi:10. 1088/0957-0233/21/11/115702

8. Kleber, X., Vincent, A.: On the role of residual internal stresses and dislocations on Barkhausen noise in plastically deformed steel. NDT\&E Int. 37, 439-445 (2004). doi:10.1016/j.ndteint.2003.11. 008
9. Makar, J.M., Tanner, B.K.: The effect of plastic deformation and residual stress on the permeability and magnetostriction of steels. J. Magn. Magn. Mater. 222, 291-304 (2000). doi:10.1016/ S0304-8853(00)00558-8

10. Kuleev, V.G., Tsar'kova, T.P., Nichipuruk, A.P.: Specific features of the behavior of the coercive force in low-carbon plastically deformed steels. Russ. J. Nondestruct. Test. 41, 285-295 (2005). doi:10.1007/s11181-005-0168-8

11. Kuleev, V.G., Tsar'kova, T.P., Sazhina, E.Y., Doroshek, A.S.: On the influence of plastic deformation of low-carbon ferromagnetic steels on the changes in the shapes of their hysteresis loops and the field dependences of the differential permeability. Russ. J. Nondestruct. Test. 51, 738-749 (2015). doi:10.1134/ S1061830915120062

12. Stupakov, O., Tomas, I., Pal'a, J., Bydzovsky, J., Bosansky, J., Smida, T.: Traditional, Barkhausen and MAT magnetic response to plastic deformation of low-carbon steel. Czech. J. Phys. 54, D47D50 (2004)

13. Samimi, A.A., Krause, T.W., Clapham, L.: Stress response of magnetic barkhausen noise in submarine hull steel: a comparative study. J. Nondestruct. Eval. 35, 32 (2016). doi:10.1007/ s10921-016-0348-6

14. Dietrich, L., Socha, G., Kowalewski, Z.L.: Anti-buckling fixture for large deformation tension-compression cyclic loading of thin metal sheets. Strain 50, 174-183 (2014). doi:10.1111/str.12078

15. Makar, J.M., Tanner, B.K.: The in situ measurement of the effect of plastic deformation on the magnetics properties of steel. Part I-Hysteresis loops and magnetostriction. J. Magn. Magn. Mater. 184, 193-208 (1998). doi:10.1016/S0304-8853(97)01129-3

16. ElBidwihy, H., Burgy, C.D., Della Torre, E.: Stress-associated changes in the magnetic properties of high strength steel. Physica B 435, 16-20 (2014). doi:10.1016/j.physb.2013.05.005

17. Hoffmann, B., Vöhringer, O., Macherauch, E.: Effect of compressive plastic deformation on mean lattice strains, dislocation densities and flow stresses of martensitically hardened steels. Mater. Sci. Eng. A 319, 299-303 (2001). doi:10.1016/ S0921-5093(01)00978-9

18. Piotrowski, L., Chmielewski, M., Augustyniak, B.: The influence of elastic deformation on the properties of the magnetoacoustic emission (MAE) signal for GO electrical steel. J. Magn. Magn. Mater. 324, 2496-2500 (2012). doi:10.1016/j.jmmm.2012.03.021

19. Langman, R.: Magnetic properties of mild steel under conditions of biaxial stress. IEEE Trans. Magn. 26(4), 1246-1251 (1990). doi: $10.1109 / 20.54015$

20. Pearson, J., Squire, P.T., Maylin, M.G., Gore, J.G.: Biaxial stress effects on the magnetic properties of pure iron. IEEE Trans. Magn. 36(5), 3251-3253 (2000)

21. Augustyniak, B.: Magneto-elastic phenomena and their applications in non-destructive evaluation of materials. Gdansk University of Technology, Gdansk (2003)

22. Hristoforou, E., Vourna, P., Ktena, A., Svec, P.: On the universality of the dependence of magnetic parameters on residual stress in steels. IEEE Trans. Magn. 52(5), 6201106 (2016). doi:10.1109/ TMAG.2015.2509642 